\title{
Pentagonal and heptagonal repdigits
}

\author{
Bir Kafle ${ }^{a}$, Florian Luca ${ }^{b}$, Alain Togbé ${ }^{a}$ \\ ${ }^{a}$ Department of Mathematics and Statistics \\ Purdue University Northwest \\ Westville, USA \\ bkafle@pnw.edu \\ atogbe@pnw.edu \\ ${ }^{b}$ School of Mathematics \\ University of the Witwatersrand \\ Wits, South Africa \\ florian.luca@wits.ac.za \\ Submitted: July 25, 2019 \\ Accepted: September 24, 2020 \\ Published online: October 8, 2020
}

\begin{abstract}
In this paper, we prove a finiteness theorem concerning repdigits represented by a fixed quadratic polynomial. We also show that the only pentagonal numbers which are also repdigits are 1,5 and 22. Similarly, the only heptagonal numbers which are repdigits are 1, 7 and 55 .
\end{abstract}

Keywords: Pentagonal numbers, heptagonal numbers, repdigits.

$M S C: 11 \mathrm{~A} 25,11 \mathrm{~B} 39,11 \mathrm{~J} 86$

\section{Introduction}

It is well known that the polygonal numbers of the forms $n(3 n-1) / 2$ and $n(5 n-3) / 2$ are called pentagonal number (OEIS [14] A000326) and heptagonal numbers (OEIS [14] A000566), respectively, where $n$ is any positive integer. Many authors have studied the problems of searching for these numbers in some interesting sequence of positive integers.

In 1996, M. Luo [6] has proved that 1 and 5 are the only pentagonal numbers in the Fibonacci sequence and later identified in [7] that 1 is the only pentagonal 
number in the Lucas sequence. The so-called generalized pentagonal numbers are given by $n(3 n-1) / 2$ with $n$ integral, not necessarily positive. In [7], again M. Luo showed that 2,1 and 7 are the only generalized pentagonal numbers which are also Lucas numbers. In [10], V. S. Rama Prasad and B. Rao proved that 1 and 7 are the only generalized pentagonal numbers in the associated Pell sequence and subsequently in [11], they identified that the only Pell numbers which are also pentagonal are $1,5,12$ and 70 .

In 2002, B. Rao [13] proved that 1, 4, 7 and 18 are the only generalized heptagonal numbers (where $n$ is any integer) in the Lucas sequence. Furthermore in [12], B. Rao identified that $0,1,13,34$ and 55 are the only generalized heptagonal numbers in the sequence of Fibonacci numbers.

A positive integer is called a repdigit (OEIS [14] A010785), if it has only one distinct digit in its decimal expansion. Repdigits have the form

$$
\ell\left(\frac{10^{m}-1}{9}\right), \quad \text { for some } m \geq 1 \text { and } \ell \in\{1,2, \ldots, 9\} \text {. }
$$

The first few repdigits are

$$
1,2,3,4,5,6,7,8,9,11,22,33,44, \ldots, 111, \ldots
$$

In addition, repunits are particular instances of repdigits, obtained when the repeating digit has the value 1 . Earlier in 2000, F. Luca [5] proved that 55 is the largest repdigit in the Fibonacci sequence, and 11 is the largest member of the Lucas sequence which is also the repdigit. In 2012, Marques and Togbé [8] studied the repdigits that are products of consecutive Fibonacci numbers.

According to Ballew and Weger [1], E. B. Escott in 1905 proved that 1, 3, 6, 55, 66 and 666 are the only triangular numbers of less than 30 digits that consist of a single repeated digit. And in 1975, they [1] proved that, in fact these are the only triangular repdigits. Recently, J. H. Jaroma [3], proved that 1 is the only integer that is both triangular and repunit.

In this paper, we first establish the finiteness of the solutions of some of the equations that involve repdigits, and consequently, we identify the petangonal and heptagonal numbers that are also repdigits.

\section{Main results}

The following result is a restatement of Theorem 1 in [4].

Theorem 2.1. Let $A, B, C$ be fixed rational numbers with $A \neq 0$. Then the Diophantine equation

$$
\ell\left(\frac{10^{m}-1}{9}\right)=A n^{2}+B n+C,
$$

has only finite number of solutions, in integers $m, n \geq 1$ and $\ell \in\{1,2, \ldots, 9\}$ provided $9 B^{2}-36 A C-4 A \ell \neq 0$. 
Proof. We multiply both sides of equation (2.1) by $4 A$, and rearrange some terms, which gives us

$$
4 A \ell\left(\frac{10^{m}-1}{9}\right)+B^{2}-4 A C=(2 A n+B)^{2} .
$$

Further, we can rewrite the last equation as

$$
4 A \ell 10^{3 m_{1}+r}+\left(9\left(B^{2}-4 A C\right)-4 A \ell\right)=9(2 A n+B)^{2},
$$

where we let $m=3 m_{1}+r$ with $r \in\{0,1,2\}$. We again multiply both sides of equation $(2.2)$ by $16 \ell^{2} 10^{2 r}$, thus we get

$$
Y^{2}=X^{3}+A
$$

where

$$
X:=4 \ell 10^{m_{1}+r}, \quad Y:=12 \ell 10^{r}(2 A n+B),
$$

and

$$
A:=16 \ell^{2} 10^{2 r}\left(9\left(B^{2}-4 A C\right)-4 A \ell\right) .
$$

By the hypothesis, we have $A \neq 0$. Thus, we obtain an elliptic curve over $\mathbb{Q}$ given by (2.3). By a theorem of Siegel (see [9], p. 313), this curve has a finite number of integer points. As a consequence, equation (2.1) has only a finite number of positive integer solutions.

The result of Ballew and Weger [1] is the case when $A=B=\frac{1}{2}$ and $C=0$ in equation (2.1), though their method of proof is different. Now, we establish some further applications of Theorem 2.1. First, we identify all the pentagonal repdigits. Our result is the following, which comes as a corollary of Theorem 2.1.

Corollary 2.2. The complete list of pentagonal repdigits is 1, 5 and 22 .

Proof. In order to prove our result, we study the equation

$$
\ell\left(\frac{10^{m}-1}{9}\right)=\frac{n(3 n-1)}{2},
$$

in integers $m, n \geq 1$ and $\ell \in\{1,2, \ldots, 9\}$, which is the case when $A=\frac{3}{2}, B=-\frac{1}{2}$ and $C=0$ in equation (2.1). Further, working as in the proof of Theorem 2.1, equation $(2.4)$ can be written as

$$
y_{1}^{2}=x_{1}^{3}+a_{1}
$$

where $x_{1}:=6 \ell 10^{m_{1}+r}, y_{1}:=9 \ell 10^{r}(6 n-1)$, and $a_{1}:=27(3-8 \ell) \ell^{2} 10^{2 r}$. We note that $a_{1}$ is nonzero, otherwise this would lead to $\ell=3 / 8$, which is not true. By Theorem 1, the equation (2.4) has only a finite number of solutions in $m, n \geq 1$ and $1 \leq \ell \leq 9$. Since $\ell \in\{1, \ldots, 9\}$ and $r \in\{0,1,2\}$, we obtain twenty-seven elliptic curves given by $(2.5)$. Now, we determine the integer points $\left(x_{1}, y_{1}\right)$ on each these elliptic curves. For this, we used MAGMA [2].

The following table displays all ${ }^{1}$ the integer points $\left(x_{1}, y_{1}\right)^{2}$, described above

\footnotetext{
${ }^{1}$ Equation $(2.5)$ has no integer points for $(\ell, r)=(1,2),(3,1),(4,1),(4,2),(5,2),(6,1),(8,1)$, $(8,2),(9,2)$.

${ }^{2}\left(x_{1}, y_{1}\right)$ 's in bold correspond to the integer solutions of the equation (2.4) in the third column.
} 
and corresponding integer solutions $(m, n)$ of the equation $(2.4)$, whenever they exist.

\begin{tabular}{|c|c|c|}
\hline$\ell, r$ & $\left(x_{1}, y_{1}\right)$ & $(m, n)$ \\
\hline $\begin{array}{l}\ell=1 \\
r=0\end{array}$ & $(6, \pm 9),(19, \pm 82),(24, \pm 117)$ & \\
\hline $\begin{array}{l}\ell=1 \\
r=1\end{array}$ & $(24, \pm 18),(\mathbf{6 0}, \pm \mathbf{4 5 0}),(85, \pm 775),(2256, \pm 107154)$ & $(1,1)$ \\
\hline $\begin{array}{l}\ell=2 \\
r=0\end{array}$ & $(12, \pm 18),(120, \pm 1314)$ & \\
\hline $\begin{array}{l}\ell=2 \\
r=1\end{array}$ & $(120, \pm 1260)$ & \\
\hline $\begin{array}{l}\ell=2 \\
r=2\end{array}$ & $\begin{array}{l}(264, \pm 2088),(300, \pm 3600),(1000, \pm 31400) \\
(\mathbf{1 2 0 0}, \pm \mathbf{4 1 4 0 0}),(24400, \pm 3811400) \\
(130296, \pm 47032344)\end{array}$ & $(2,4)$ \\
\hline $\begin{array}{l}\ell=3 \\
r=0\end{array}$ & $(18, \pm 27),(288, \pm 4887)$ & \\
\hline $\begin{array}{l}\ell=3 \\
r=2\end{array}$ & $(856, \pm 24004)$ & \\
\hline $\begin{array}{l}\ell=4 \\
r=0\end{array}$ & $\begin{array}{l}(24, \pm 36),(33, \pm 153),(112, \pm 1180),(384, \pm 7524) \\
(528, \pm 12132)\end{array}$ & \\
\hline $\begin{array}{l}\ell=5 \\
r=0\end{array}$ & $\begin{array}{l}(30, \pm 45),(46, \pm 269),(64, \pm 487),(75, \pm 630), \\
(120, \pm 1305),(480, \pm 24345),(1654, \pm 67267)\end{array}$ & \\
\hline $\begin{array}{l}\ell=5 \\
r=1\end{array}$ & $\begin{array}{l}(136, \pm 134),(\mathbf{3 0 0}, \pm \mathbf{4 9 5 0}),(525, \pm 11925) \\
(4800, \pm 332550)\end{array}$ & $(1,2)$ \\
\hline $\begin{array}{l}\ell=6 \\
r=0\end{array}$ & $(36, \pm 54),(1224, \pm 42822)$ & \\
\hline $\begin{array}{l}\ell=6 \\
r=2\end{array}$ & $(1224, \pm 37368)$ & \\
\hline $\begin{array}{l}\ell=7 \\
r=0\end{array}$ & $(42, \pm 63),(1680, \pm 68859)$ & \\
\hline $\begin{array}{l}\ell=7 \\
r=1\end{array}$ & $\begin{array}{l}(240, \pm 2610),(301, \pm 4501),(420, \pm 8190), \\
(2940, \pm 159390)\end{array}$ & \\
\hline $\begin{array}{l}\ell=7 \\
r=2\end{array}$ & $(4200, \pm 270900)$ & \\
\hline $\begin{array}{l}\ell=8 \\
r=0\end{array}$ & $(48, \pm 72),(2208, \pm 103752)$ & \\
\hline $\begin{array}{l}\ell=9 \\
r=0\end{array}$ & $\begin{array}{l}(54, \pm 81),(108, \pm 1053),(162, \pm 2025),(279, \pm 4644) \\
(2808, \pm 148797),(2979, \pm 162594), \\
(3310254, \pm 6022710369)\end{array}$ & \\
\hline $\begin{array}{l}\ell=9 \\
r=1\end{array}$ & $(1296, \pm 46494)$ & \\
\hline
\end{tabular}

Table 1: Integer solutions $\left(x_{1}, y_{1}\right)$ 
The list of ordered pair $(m, n)$ in third column of Table 1 above, together with the corresponding values of $\ell$ in the first column give us the complete list of the solutions $(m, n, \ell)$ in positive integers for equation (2.4). From this, we can deduce that the only pentagonal numbers in the sequence of repdigits are given by the statement of Corollary 2.2. This completes the proof of Corollary 2.2.

Next, we identify all the heptagonal numbers in the sequence of the repdigits. Our result is the following.

Corollary 2.3. The complete list of heptagonal repdigits is 1, 7 and 55 .

Proof. We let $A=\frac{5}{2}, B=-\frac{3}{2}$ and $C=0$ in equation (2.1), which allows us to study the following equation (finite number of solutions, by Theorem 2.1),

$$
\ell\left(\frac{10^{m}-3}{9}\right)=\frac{n(5 n-1)}{2},
$$

in integers $m, n \geq 1$ and $\ell \in\{1,2, \ldots, 9\}$. As before, last equation can be reduced to

$$
y_{2}^{2}=x_{2}^{3}+a_{2},
$$

where $x_{2}:=10 \ell 10^{m_{1}+r}, y_{2}:=15 \ell 10^{r}(10 n-3)$, and $a_{2}:=25 \ell^{2} 10^{2 r}(81-40 \ell)$. We note that $a_{2}$ is nonzero, otherwise we get $\ell=81 / 40$, which is not true. Now, we use MAGMA [2], to determine the integer points $\left(x_{2}, y_{2}\right)$ on the elliptic curves given by $(2.7)$.

The following table shows all ${ }^{3}$ the integer points $\left(x_{2}, y_{2}\right)^{4}$, described above and corresponding integer solutions $(m, n)$ of the equation $(2.6)$, whenever they exist.

\begin{tabular}{|l|l|l|}
\hline$\ell, r$ & $\left(x_{2}, y_{2}\right)$ & $(m, n)$ \\
\hline$\ell=1$, & $\begin{array}{l}(10, \pm 5),(5, \pm 30),(4, \pm 31),(1, \pm 32),(4, \pm 33), \\
(10, \pm 45),(20, \pm 95),(40, \pm 225),(50, \pm 355),(64, \pm 513), \\
(155, \pm 1930),(166, \pm 2139),(446, \pm 9419),(920, \pm 27905), \\
(3631, \pm 218796),(3730, \pm 227805)\end{array}$ & \\
\hline $\begin{array}{l}\ell=1, \\
r=1\end{array}$ & $(\mathbf{1 0 0}, \pm \mathbf{1 0 5 0})$ & $(1,1)$ \\
\hline $\begin{array}{l}\ell=1, \\
r=2\end{array}$ & $(200, \pm 1500),(2000, \pm 89500)$ & \\
\hline $\begin{array}{l}\ell=2, \\
r=0\end{array}$ & $\begin{array}{l}(4, \pm 6),(0, \pm 10),(5, \pm 15),(20, \pm 90),(24, \pm 118), \\
(2660, \pm 137190)\end{array}$ & \\
\hline $\begin{array}{l}\ell=2, \\
r=1\end{array}$ & $(0, \pm 100)$ & \\
\hline $\begin{array}{l}\ell=2, \\
r=2\end{array}$ & $(100, \pm 0),(0, \pm 1000),(200, \pm 3000)$ & \\
\hline
\end{tabular}

\footnotetext{
${ }^{3}$ Equation (2.7) has no integer points for $(\ell, r)=(6,1),(6,2),(8,1),(9,1)$.

${ }^{4}\left(x_{2}, y_{2}\right)$ 's in bold correspond to the integer solutions of the equation (2.6) in the third column.
} 


\begin{tabular}{|c|c|c|}
\hline $\begin{array}{l}\ell=3 \\
r=0\end{array}$ & $(30, \pm 135),(40, \pm 235),(1299, \pm 46818)$ & \\
\hline $\begin{array}{l}\ell=3 \\
r=1\end{array}$ & $(100, \pm 350)$ & \\
\hline $\begin{array}{l}\ell=3 \\
r=2\end{array}$ & $(1200, \pm 40500)$ & \\
\hline $\begin{array}{l}\ell=4 \\
r=0\end{array}$ & $(40, \pm 180)$ & \\
\hline $\begin{array}{l}\ell=4 \\
r=1\end{array}$ & $\begin{array}{l}(184, \pm 1752),(200, \pm 2200),(400, \pm 7800),(1900, \pm 82800), \\
(60625, \pm 14927175)\end{array}$ & \\
\hline $\begin{array}{l}\ell=4 \\
r=2\end{array}$ & $(800, \pm 14000)$ & \\
\hline $\begin{array}{l}\ell=5 \\
r=0\end{array}$ & $(50, \pm 225),(134, \pm 1527),(7550, \pm 656025)$ & \\
\hline $\begin{array}{l}\ell=5 \\
r=1\end{array}$ & $(200, \pm 750),(6000, \pm 464750)$ & \\
\hline $\begin{array}{l}\ell=5 \\
r=2\end{array}$ & $(5000, \pm 352500)$ & $(2,5)$ \\
\hline $\begin{array}{l}\ell=6 \\
r=0\end{array}$ & $(60, \pm 270),(280, \pm 4670)$ & \\
\hline $\begin{array}{l}\ell=7 \\
r=0\end{array}$ & $(70, \pm 315),(91, \pm 714),(200, \pm 2785),(2240, \pm 106015)$ & \\
\hline $\begin{array}{l}\ell=7 \\
r=1\end{array}$ & $\begin{array}{l}(301, \pm 1701),(\mathbf{7 0 0}, \pm \mathbf{1 7 8 5 0}),(1400, \pm 52150) \\
(7900, \pm 702150)\end{array}$ & $(1,2)$ \\
\hline $\begin{array}{l}\ell=7 \\
r=2\end{array}$ & $\begin{array}{l}(1400, \pm 17500),(25424, \pm 4053532),(49000, \pm 10846500) \\
(325000, \pm 185278500)\end{array}$ & \\
\hline $\begin{array}{l}\ell=8 \\
r=0\end{array}$ & $\begin{array}{l}(80, \pm 360),(120, \pm 1160),(200, \pm 2760),(396, \pm 7856) \\
(1244, \pm 43872),(2081, \pm 94929)\end{array}$ & \\
\hline $\begin{array}{l}c=8 \\
r=2\end{array}$ & $(1700, \pm 33000),(2400, \pm 100000),(32000,5724000)$ & \\
\hline $\begin{array}{l}\ell=9 \\
r=0\end{array}$ & $\begin{array}{l}(90, \pm 405),(171, \pm 2106),(180, \pm 2295),(630, \pm 15795) \\
(700, \pm 18505),(720, \pm 19305), \\
(150750, \pm 58531005),(238770, \pm 116672805)\end{array}$ & \\
\hline $\begin{array}{l}\ell=9 \\
r=2\end{array}$ & $\begin{array}{l}(1800, \pm 13500),(2016, \pm 50436),(3600, \pm 202500) \\
(5625, \pm 415125),(9000, \pm 850500),(25425, \pm 4053375) \\
(83800, \pm 24258500),(126000, \pm 44725500)\end{array}$ & \\
\hline
\end{tabular}

Table 2: Integer solutions $\left(x_{2}, y_{2}\right)$

In Table 2, as in the proof of Corollary 2.2, the list of ordered pair $(m, n)$ in third column together with the corresponding values of $\ell$ in the first column give us the complete list of the solutions $(m, n, \ell)$ in positive integers with $1 \leq \ell \leq 9$ for the equation (2.4), which are the only pentagonal numbers in the sequence of repdigits. This completes the proof of Corollary 2.3 . 
Recently in [4], authors of this paper studied the triangular numbers that are also repeated blocks of two digits, which we call the repblocks of two digits. Such numbers have the form

$$
\ell\left(\frac{10^{2 m}-1}{99}\right), \quad \text { for some } m \geq 1 \text { and } \ell \in\{10,11, \ldots, 99\} .
$$

Additionally in this paper, we extend and complement the results obtained in [4] by finding all the pentagonal repblocks of two digits. Our results are the following.

Corollary 2.4. The complete list of pentagonal numbers which are also repblocks of two digits is

$$
12,22,35,51,70,92,1717 .
$$

Proof. To prove our result, in equation (2.1), we replace the left hand side by $\ell\left(\frac{10^{2 m}-1}{99}\right)$, with $\ell \in\{10,11, \ldots, 99\}$ and the right hand side of it by $A=\frac{3}{2}, B=$ $-\frac{1}{2}$ and $C=0$. As before, the resulting equation can be written as

$$
y_{3}^{2}=x_{3}^{3}+a_{3}
$$

where $x_{3}:=66 \ell 10^{2 m_{1}+2 r}, y_{3}:=1089 \ell 10^{2 r}(6 n-1)$ and $a_{3}:=11979 \ell^{2} 10^{4 r}(3-8 \ell)$. We note that $a_{3}$ is nonzero. Now, we use MAGMA [2], to determine the integer points $\left(x_{3}, y_{3}\right)$ on the two hundred forty-three elliptic curves given by (2.8).

The following table displays all the integer points $\left(x_{3}, y_{3}\right)^{5}$ of $(2.8)$, which produce the corresponding integer solutions $(m, n)$ of equation $(2.4)$. There are only seven such elliptic curves. The other two hundred thirty-six equations either do not have any integer points $\left(x_{3}, y_{3}\right)$, or do not produce relevant solutions $(m, n)$ and thus, we omit those equations.

\begin{tabular}{|l|l|l|}
\hline$\ell, r$ & $\left(x_{3}, y_{3}\right)$ & $(m, n)$ \\
\hline$\ell=12$, & $(25524, \pm 3656232),(\mathbf{7 9 2 0 0}, \pm \mathbf{2 2 2 1 5 6 0 0})$, & $(1,3)$ \\
$r=1$ & $(127600, \pm 45544400),(1753200, \pm 2321384400)$ & \\
\hline$\ell=17$, & $(1734000, \pm 2259810000),(3706000, \pm 7126910000)$, & \\
$\quad(4686000, \pm 10138590000),(\mathbf{1 1 2 2 0 0 0 0}, \pm \mathbf{3 7 5 8 1 3 9 0 0 0 0})$, & $(2,34)$ \\
$r=2$ & $(17217600, \pm 71442126000),(20476500, \pm 92657565000)$, & \\
\hline $\begin{array}{l}(166268400, \pm 2143949598000) \\
\ell=22,\end{array}$ & $\begin{array}{l}(31944, \pm 2779128),(36300, \pm 4791600), \\
(121000, \pm 41793400),(\mathbf{1 4 5 2 0 0}, \pm \mathbf{5 5 1 0 3 4 0 0}),\end{array}$ & $(2,4)$ \\
\hline $\begin{array}{l}\ell=35, \\
r=1\end{array}$ & $(2952400, \pm 5072973400),(15765816, \pm 62600049864)$ & \\
\hline$\ell=51$, & $(279400, \pm 13954500),(\mathbf{2 3 1 0 0 0}, \pm \mathbf{1 1 0 5 3 3 5 0 0})$, & $(2,5)$ \\
$r=1$ & $(\mathbf{3 3 6 6 0 0}, \pm \mathbf{1 9 4 3 8 6 5 0 0})$ & $(1,6)$ \\
\hline
\end{tabular}

\footnotetext{
${ }^{5}\left(x_{3}, y_{3}\right)$ 's in bold correspond to the integer solutions in the third column.
} 


\begin{tabular}{|c|c|c|}
\hline $\begin{array}{l}\ell=70 \\
r=1\end{array}$ & $(462000, \pm 312543000)$ & $(1,7)$ \\
\hline $\begin{array}{l}\ell=92 \\
r=1\end{array}$ & $(607200, \pm 470883600)$ & $(1,8)$ \\
\hline
\end{tabular}

Table 3: Integer solutions $\left(x_{3}, y_{3}\right)$

The ordered pairs $(m, n)$ in the third column of Table 3, together with the corresponding values of $\ell$ in the first column give us the complete list of pentagonal numbers which are also the repblock of two digits. This completes the proof of Corollary 2.4.

In the same fashion, one can show that 18, 34, 55, 81 and 4141 are the only heptagonal numbers which are also repblocks of two digits.

Acknowledgements. B. K. and A. T. are partially supported by Purdue University Northwest, IN.

\section{References}

[1] D. W. Ballew, R. C. Weger: Repdigit triangular numbers, J. Recreational Math. 8.2 (1975-1976), pp. 96-97.

[2] J. Cannon, C. Playoust: MAGMA: a new computer algebra system, Euromath Bull. 2.1 (1996), pp. 113-144.

[3] J. H. Jaroma: Triangular repunit - there is but 1, Czech. Math. J. 60 (2010), pp. 1075-1077, DOI: https://doi.org/10.1007/s10587-010-0072-9.

[4] B. Kafle, F. Luca, A. Togbé: Triangular repblocks, The Fibonacci Quart. 56.4 (2018), pp. 325-328.

[5] F. LucA: Fibonacci and Lucas numbers with only one distinct digit, Portugaliae Math. 57.2 (2000), pp. 243-254.

[6] M. Luo: Pentagonal numbers in the Fibonacci sequence, Appl. Fibonacci Numbers, Eds. G. E. Bergum et al., Kluwer 6 (1996), pp. 349-354, DOI: https://doi.org/10.1007/978-94-009-0223-7_29.

[7] M. Luo: Pentagonal numbers in the Lucas sequence, Portugaliae Math. 53.3 (1996), pp. 325329.

[8] D. Marques, A. Togbé: On repdigits as product of consecutive Fibonacci numbers, Rend. Istit. Mat. Univ. Trieste 44 (2012), pp. 393-397.

[9] R. A. Mollin: Advanced Number Theory with Applications, Boca Raton, FL: CRC Press, 2010 DOI: https://doi.org/10.1201/b12331.

[10] V. S. R. Prasad, B. S. RaO: Pentagonal numbers in the Associated Pell sequence and Diophantine equations $x^{2}(3 x-1)^{2}=8 y^{2} \pm 2$, The Fibonacci Quart. 39.4 (2001), pp. 299-303.

[11] V. S. R. Prasad, B. S. Rao: Pentagonal numbers in the Pell sequence and Diophantine equations $2 x^{2}=y^{2}(3 y-1)^{2} \pm 2$, The Fibonacci Quart. 40.3 (2002), pp. 233-241. 
[12] B. S. RAO: Heptagonal numbers in the Fibonacci sequence and Diophantine equations $4 x^{2}=$ $5 y^{2}(5 y-3)^{2} \pm 16$, The Fibonacci Quart. 41.5 (2003), pp. 414-420.

[13] B. S. RAO: Heptagonal numbers in the Lucas sequence and Diophantine equations $x^{2}(5 x-$ $3)^{2}=20 y^{2} \pm 16$, The Fibonacci Quart. 40.4 (2002), pp. 319-322.

[14] N. J. A. Slonne: The Online Encyclopedia of Integer Sequences, OEIS Foundation Inc., electronically at http://oeis.org (2020). 